\title{
Einleitung: Zwanzig Jahre Deutscher Freiwilligensurvey
}

Julia Simonson, Nadiya Kelle, Corinna Kausmann \&

Clemens Tesch-Römer

\section{Kernaussagen}

Der Freiwilligensurvey ist seit zwei Jahrzehnten die Basis für die Berichterstattung zum aktuellen Stand und zur Entwicklung des freiwilligen Engagements in Deutschland. Seit 1999 wird er alle fünf Jahre als telefonische bevölkerungsrepräsentative Studie durchgeführt. Aktuell liegen die Daten der fünften Erhebung aus dem Jahr 2019 vor.

Als freiwilliges Engagement werden Tätigkeiten gefasst, die freiwillig und gemeinschaftsbezogen ausgeübt werden, im öffentlichen Raum stattfinden und nicht auf materiellen Gewinn gerichtet sind. Die im Freiwilligensurvey verwendete Definition des Engagements deckt sich somit weitgehend mit der von der Enquete-Kommission "Zukunft des Bürgerschaftlichen Engagements“ im Jahr 2002 entwickelten Begriffsbestimmung bürgerschaftlichen Engagements.

Im öffentlichen Diskurs wird die hohe gesellschaftspolitische Bedeutung freiwilligen Engagements hervorgehoben; allerdings ist freiwilliges Engagement nicht in jedem Fall förderlich für die Gesellschaft. Häufig wird argumentiert, dass freiwilliges Engagement zum Zusammenhalt der Gesellschaft beitragen und positive Auswirkungen für die Zielgruppen des Engagements und für die Engagierten selbst haben kann. Allerdings kann sich Engagement auch als problematisch für die Gesellschaft erweisen, beispielsweise dann, wenn in der freiwilligen Tätigkeit demokratiefeindliche Ziele verfolgt werden. 
Der gesellschaftliche Wandel findet durch neu aufgenommene Themen Eingang in den Freiwilligensurvey. Die gesellschaftlichen Veränderungen der letzten zwei Jahrzehnte schaffen Potenziale für neue Engagementformen und wirken sich auf die Ausgestaltung zivilgesellschaftlichen Handelns aus. Dementsprechend wurden im Jahr 2019 erstmals Fragen zu Einstellungen zur Demokratie, zum Engagement von Geflüchteten und für Geflüchtete, zu Formen der Internetnutzung im Rahmen der freiwilligen Tätigkeit sowie zu den Zwecken von Geldspenden gestellt.

Freiwilliges Engagement ist vielfältig und voraussetzungsvoll. Inhalte und Formen des Engagements unterscheiden sich ebenso wie die Engagierten selbst. Um Ungleichheiten im Zugang zum Engagement zu untersuchen, die beispielsweise daraus resultieren, dass nicht alle Menschen über die gleichen Ressourcen für eine freiwillige Tätigkeit verfügen, müssen Unterschiede zwischen Bevölkerungsgruppen berücksichtigt werden.

Um die Bevölkerung mit dem Freiwilligensurvey noch besser als bisher abbilden zu können, wurde im Jahr 2019 die Gewichtung um das Merkmal der schulischen Bildung ergänzt. Dieses Vorgehen hat zur Folge, dass die Engagementquoten aller Erhebungsjahre um drei bis vier Prozentpunkte niedriger ausfallen als die bislang ohne diese Gewichtung berechneten Quoten. Bei der Betrachtung der Engagementquoten über die Zeit ändert sich somit das Niveau, der Trend bleibt jedoch weitgehend bestehen. 
Freiwilliges Engagement ist eine wichtige Säule der Gesellschaft. Im öffentlichen Diskurs wird die gesellschaftspolitische Bedeutung freiwilligen Engagements hervorgehoben. Freiwilliges Engagement kann zum Zusammenhalt der Gesellschaft beitragen und ist unverzichtbar für eine gelingende und lebendige Demokratie (Roth 2010; Verba et al. 1995). Freiwilliges Engagement ist vielfältig: Menschen engagieren sich, um sich für ein Anliegen wie Menschenrechte oder Klimaschutz einzusetzen, sie bringen sich in den öffentlichen Diskurs ein, sie gestalten ihr lokales Umfeld und sie nehmen Einfluss auf politische Entscheidungen. Häufig geht es beim freiwilligen Engagement auch um die Solidarität mit anderen Menschen wie etwa bei der Unterstützung von Geflüchteten oder hilfebedürftigen Menschen. Freiwilliges Engagement umfasst schließlich auch die Gestaltung des gemeinsamen Miteinanders, zum Beispiel in der Elternvertretung der Schule oder im Sportverein durch die Leitung von Trainingseinheiten.

Für Gesellschaft und Politik ist es bedeutsam, wie sich freiwilliges Engagement über die Zeit hinweg entfaltet: Nimmt der Anteil freiwillig engagierter Menschen in der Gesellschaft zu - oder ab? Verändert sich die Art des Engagements? Investieren freiwillig engagierte Menschen mehr oder weniger Zeit in ihr Engagement? Steigt oder sinkt die Bereitschaft längerfristige Aufgaben wie Leitungsfunktionen in zivilgesellschaftlichen Organisationen zu übernehmen? Unterscheiden sich Ausmaß und Art des Engagements zwischen Frauen und Männern, zwischen jüngeren und älteren Menschen, zwischen Menschen mit und ohne Migrationshintergrund - und haben sich Unterschiede zwischen diesen Gruppen vergrößert oder verkleinert? Abhängig von den jeweiligen Entwicklungen ist es sinnvoll, organisationale und politische Rahmenbedingungen an gewandelte Formen von Engagement anzupassen.

Der Deutsche Freiwilligensurvey (FWS), vom Bundesministerium für Familie, Senioren, Frauen und Jugend (BMFSFJ) ins Leben gerufen und gefördert, ermöglicht die Beantwortung dieser und weiterer Fragen. Der Freiwilligensurvey ist eine repräsentative telefonische Befragung zum freiwilligen Engagement in Deutschland, die seit 1999 alle fünf Jahre durchgeführt wird und sich an Personen ab 14 Jahren richtet. Er ist seit zwei Jahrzehnten die Basis für die Berichterstattung zur Situation und Entwicklung des freiwilligen Engagements in Deutschland.

Anhand der aktuellen Daten des Freiwilligensurveys 2019 lässt sich die Entwicklung des freiwilligen Engagements im Zeitvergleich nachzeichnen. Es lässt sich weiterhin überprüfen, welche Unterschiede es hinsichtlich der Beteiligung im Engagement zwischen Bevölkerungsgruppen gibt und wie unterschiedlich freiwilliges Engagement ausgestaltet sein kann. Darüber hinaus ermöglicht der Freiwilligensurvey Aussagen über die Motive und Hinderungsgründe für freiwilliges Engagement sowie die Bereitschaft von derzeit nicht-engagierten Personen, sich in Zukunft zu engagieren. Zusätzlich stellt der Freiwilligensurvey 2019 Informationen zu weiteren Aspekten des zivilgesellschaftlichen Handelns wie dem Spendenverhalten oder der politischen Partizipation sowie zu Einstellungen der Menschen zur Demokratie und gesellschaftlichen Institutionen bereit. 


\subsection{Was ist freiwilliges Engagement?}

\section{Konzeptuelle Definition freiwilligen Engagements}

Im Freiwilligensurvey wird eine weite Definition des freiwilligen Engagements verwendet. ${ }^{1}$ Der Begriff des ,freiwilligen Engagements ${ }^{c}$ dient als Oberbegriff für unterschiedliche Formen des Engagements, das von selbstorganisierten und/oder eher sporadisch ausgeübten Tätigkeiten bis zu Freiwilligendiensten reicht, die sich durch eine gesetzlich geregelte Organisationsform und feste Einsatzzeiten auszeichnen.

Die im Freiwilligensurvey verwendete Definition des Engagements deckt sich mit den von der Enquete-Kommission "Zukunft des Bürgerschaftlichen Engagements" im Jahr 2002 entwickelten Kriterien zur Begriffsbestimmung bürgerschaftlichen Engagements (Deutscher Bundestag 2002: 32). Laut Enquete-Kommission ist eine Tätigkeit dann als bürgerschaftliches Engagement zu bezeichnen, wenn sie die folgenden fünf Kriterien erfüllt (Deutscher Bundestag 2002: 38 ff.):

- Die Tätigkeit ist freiwillig: Das Engagement beruht auf einer eigenen, freiwilligen Entscheidung.

- Die Tätigkeit ist öffentlich beziehungsweise findet im öffentlichen Raum statt: Damit ist das Engagement abgrenzbar von Tätigkeiten, die im familiären oder beruflichen Rahmen ausgeübt werden.

- Die Tätigkeit wird in der Regel gemeinschaftlich/kooperativ ausgeübt: Der Bezug zur Gemeinschaft meint hierbei zum einen „das Wohl der Mitglieder einer mehr oder weniger umfassenden Gruppe von Gesellschaftsmitgliedern" und zum anderen die Tätigkeit selbst, welche in der Regel eine „Tätigkeit mit anderen“ darstellt (Deutscher Bundestag 2002: 39).

- Die Tätigkeit ist nicht auf materiellen Gewinn gerichtet: Sie dient nicht dazu, ein monetäres Einkommen zu erzielen oder den eigenen Nutzen zu erhöhen. Ein immaterieller persönlicher Nutzen durch das Ausüben einer als sinnvoll empfundenen Tätigkeit kann aber durchaus angestrebt werden.

- Die Tätigkeit ist gemeinwohlorientiert: Da unterschiedliche Auffassungen darüber bestehen, was zum Gemeinwohl beiträgt, wird in der Regel die Intention der handelnden Personen in den Blick genommen. Gemeinwohlorientierung und Eigeninteresse können dabei durchaus Hand in Hand gehen: Ein Engagement kann sowohl den Interessen der engagierten Person als auch dem Gemeinwohl dienen.

1 Für einen Überblick zu unterschiedlichen Begriffsdefinitionen siehe Kausmann et al. 2019. 


\section{Erfassung des freiwilligen Engagements im Freiwilligensurvey}

Die Kriterien der Enquete-Kommission werden im Freiwilligensurvey berücksichtigt und finden Eingang in die Fragen zum freiwilligen Engagement im Interview sowie in die Prüfung der offenen Angaben zu den freiwilligen Tätigkeiten der Befragten. Allerdings können nicht alle von der Enquete-Kommission festgelegten Kriterien im Rahmen einer empirischen Erfassung operationalisiert werden.

Die empirische Erfassung des freiwilligen Engagements beruht seit Beginn des Freiwilligensurveys im Jahr 1999 auf Selbstangaben der befragten Personen. Freiwilliges Engagement wird dazu zweistufig erfasst. ${ }^{2}$ Im ersten Schritt werden Aktivitäten in vierzehn gesellschaftlichen Bereichen außerhalb von Beruf und Familie in den letzten zwölf Monaten erfragt. Im zweiten Schritt wird ermittelt, ob die Person in den von ihr genannten Bereichen in den letzten zwölf Monaten auch freiwillig und unentgeltlich oder gegen geringe Aufwandsentschädigung Aufgaben oder Arbeiten übernommen hat.

Die so erfassten Tätigkeiten spiegeln das Alltagsverständnis freiwilligen Engagements der Befragten wider, können aber von der oben dargelegten konzeptuellen Definition freiwilligen Engagements abweichen. Daher wird in mehreren Schritten geprüft, ob eine Tätigkeit gegen die Kriterien der konzeptuellen Definition verstößt. Während des Interviews werden alle Personen, die angegeben haben, eine Aufgabe übernommen zu haben, in standardisierter Form noch einmal gefragt, ob es sich dabei wirklich um eine ehrenamtliche oder freiwillige, unbezahlte oder gegen geringe Aufwandsentschädigung ausgeübte Tätigkeit handelt. Nur wenn eine Person diese Nachfrage bejaht, gilt sie für den weiteren Interviewverlauf als engagiert und es werden weitere Fragen zum Engagement gestellt. Nach der Datenerhebung erfolgt eine weitere Prüfung anhand der offenen Angaben aus drei Abfragen des Interviews:

- Um welche Art von Gruppe, Organisation oder Einrichtung handelt es sich, in der Sie tätig sind?

- Was machen Sie dort konkret? Welche Aufgabe oder Arbeit üben Sie dort aus?

- Hat Ihre Tätigkeit einen Namen? Wenn ja, welchen?

Diese Selbstangaben der Personen zu ihren freiwilligen Tätigkeiten werden im Anschluss an die Datenerhebung anhand eines Kriterienkatalog überprüft. Die Kriterien umfassen die aktive Übernahme einer Tätigkeit, die Unentgeltlichkeit, die Öffentlichkeit sowie die Gemeinschaftlichkeit der Tätigkeit (siehe hierzu Kapitel 2). Angaben, die eindeutig gegen mindestens eines der genannten Kriterien verstoßen, werden nicht als freiwillige Tätigkeit gewertet und fließen nicht in die Berechnung der Engagementquote ein.

2 Siehe zur Erfassung des freiwilligen Engagements auch Kapitel 3. 
Das Kriterium der Freiwilligkeit wird bereits während des Interviews im Rahmen der standardisierten Nachfrage geprüft. Darüber hinaus gibt es im Jahr 2019 keine verpflichtenden Dienste in Deutschland, sodass auf eine systematische Prüfung anhand der offenen Angaben verzichtet werden kann.

Das Kriterium der Gemeinwohlorientierung wird in allen Erhebungen des Freiwilligensurveys seit 1999 nicht überprüft. Der Begriff der Gemeinwohlorientierung kann von Personen mit unterschiedlichen Norm- und Wertevorstellungen sehr unterschiedlich, ja konträr verstanden werden. Eine Tätigkeit, die vor dem Hintergrund eines bestimmten Wertesystems als, gemeinwohlorientiert' verstanden wird, kann vor dem Hintergrund eines konkurrierenden Wertesystems als ,gemeinwohlschädigend' interpretiert werden. Darüber hinaus ist davon auszugehen, dass eine Abfrage der Gemeinwohlorientierung starken Effekten sozialer Erwünschtheit unterliegen würde. Die Gemeinwohlorientierung des Engagements wurde daher weder in der Erhebungswelle 2019, noch in den davorliegenden Erhebungsjahren geprüft; sie wurde vielmehr seit der ersten Welle des Freiwilligensurveys als gegeben vorausgesetzt.

\subsection{Gesellschaftspolitische Bewertung des freiwilligen Engagements}

In der öffentlichen Debatte wird freiwilliges Engagement ganz überwiegend positiv bewertet. Es kann als wichtige Form der sozialen Teilhabe für das Individuum, das sich engagiert, gesehen werden (Simonson \& Vogel 2020) und sich nicht nur positiv auf die soziale Einbindung und das Ansehen der Engagierten, sondern auch auf ihre Gesundheit und Lebenszufriedenheit auswirken (zum Beispiel Morrow-Howell et al. 2009; Wilson 2012). Freiwilliges Engagement kann darüber hinaus anderen Personen zugutekommen, die als Zielgruppen des Engagements adressiert werden. Schließlich kann es als eine der zentralen Formen gesellschaftlicher Partizipation zum Zusammenhalt der Gesellschaft beitragen (Alscher et al. 2021) und die Demokratie stärken (Ebert 2018). Ein wichtiger Aspekt ist dabei die Gemeinwohlorientierung freiwilligen Engagements. Was als Gemeinwohl zu verstehen ist, ist in der Regel das Ergebnis von Abstimmungs- und Aushandlungsprozessen, denn in einer Gesellschaft können durchaus unterschiedliche Auffassungen darüber bestehen, was dem Gemeinwohl dient und was nicht (Deutscher Bundestag 2002). So gibt es auch freiwilliges Engagement, das zwar aus individueller Perspektive der engagierten Personen gemeinwohlorientiert ist, der Demokratie und der Gesellschaft aber letztlich eher schadet als nützt, beispielsweise wenn demokratiefeindliche Ziele verfolgt werden oder das Engagement die Ausgrenzung einzelner Bevölkerungsgruppen anstrebt (Klein \& Müller 2016).

Schließlich kann freiwilliges Engagement nur dann eine Ressource für demokratische Prozesse sein, wenn es auf demokratischen Prinzipien beruht und demokratische Ziele verfolgt (Geißel 2006; Putnam 200o). Bei einem Engagement, das zum Ziel hat, die Demokratie zu schwächen, zu beschädigen oder sogar abzuschaffen, wird die 
Erwartung, dass freiwilliges Engagement den gesellschaftlichen Zusammenhalt stärkt und die Demokratie festigt, dagegen nicht erfüllt.

Eine Annäherung daran, wie unterschiedlich sich die Perspektive der Menschen darstellen kann, soll mithilfe der neuen Fragen zu Einstellungen zur Demokratie und zum Vertrauen in staatliche Institutionen geschaffen werden, mit denen Auskunft darüber gegeben werden kann, wie positive und negative Einstellungen zur Demokratie und zu deren Institutionen bei engagierten und nicht-engagierten Menschen in Deutschland verteilt sind.

\subsection{Gesellschaftlicher Wandel und freiwilliges Engagement}

Freiwilliges Engagement und seine Ausgestaltung stehen in engem Zusammenhang mit den gesellschaftlichen Rahmenbedingungen und deren Wandel über die Zeit. Zwischen 1999 und 2019 hat es eine Reihe unterschiedlicher gesellschaftlicher Entwicklungen gegeben, die als Rahmenbedingungen für das freiwillige Engagement in Deutschland bedeutsam sind:

- Öffentliche Thematisierung des freiwilligen Engagements und Wandel der Engagementpolitik: Freiwilliges Engagement hat als Thema in der politischen und öffentlichen Debatte in den letzten Jahren deutlich an Aufmerksamkeit gewonnen. Zugleich hat sich die Engagementpolitik als eigenes Politikfeld herausgebildet (Hartnuß et al. 2011).

- Veränderungen der Strukturen für freiwilliges Engagement: Die Zahl der Stiftungen und Vereine ist in den letzten Jahrzehnten deutlich gestiegen, allerdings nach 2014 nicht mehr so stark wie in den Jahren zuvor (Priemer et al. 2019). Dies hat Auswirkungen auf die Gelegenheitsstrukturen für freiwilliges Engagement.

- Veränderungen in Richtung einer Gleichstellung von Frauen und Männern: In den vergangenen Jahrzehnten hat sich der politische und gesellschaftliche Diskurs zur Gleichstellung der Geschlechter weiterentwickelt (Deutscher Bundestag 2017; Sellach \& Libuda-Köster 2017). Auch wenn es bislang noch keine umfassende Gleichstellung von Frauen und Männern in allen Lebensbereichen gibt, können bereits die gesellschaftliche Debatte darüber und die Entwicklungen in Richtung einer Gleichstellung zu einer Veränderung der Beteiligung von Frauen und Männern am freiwilligen Engagement geführt haben.

- Bildungsexpansion: Der Anteil von Menschen mit höherer Bildung in der Bevölkerung hat sich in den letzten Jahrzehnten stetig erhöht (Blaeschke \& Freitag 2021). Da sich Menschen mit höherer Bildung zu größeren Anteilen freiwillig engagieren als Menschen mit niedrigerem Bildungsniveau, ist davon auszugehen, dass sich dies steigernd auf die Beteiligung im Engagement auswirkt.

- Gestiegene Erwerbsbeteiligung: Die Erwerbsbeteiligung insbesondere von Frauen sowie von älteren Menschen ist gestiegen (Wagner 2017; Rauch et al. 2017). Diese 
Entwicklung kann sich auch im Engagement niederschlagen, da sich über die Erwerbsarbeit zusätzliche Gelegenheiten für freiwilliges Engagement ergeben können. Andererseits kann die geringere zur Verfügung stehende Zeit auch dazu führen, dass seltener ein freiwilliges Engagement ausgeübt wird.

- Veränderungen der Lebensphase Alter: Die heute Älteren haben durch die gestiegene Lebenserwartung die Aussicht auf eine viele Jahre andauernde Phase des beruflichen Ruhestandes, die sie im Durchschnitt bei vergleichsweise guter Gesundheit verbringen können. Die heute Älteren sind außerdem im Durchschnitt höher gebildet als die Älteren früherer Geburtsjahrgänge. Hinzu kommt ein politischer Diskurs des aktiven Alterns, durch den freiwilliges Engagement gefördert wird (Simonson \& Vogel 2020; Vogel et al. 2019).

- Zuwanderungsentwicklung: Personen, die nach Deutschland einwandern, bringen das Potenzial mit sich, sich selbst freiwillig zu engagieren. Ebenso werden sie durch das freiwillige Engagement anderer unterstützt. Insbesondere im Zusammenhang mit der im Jahr 2015 gestiegenen Zuwanderung geflüchteter Menschen nach Deutschland kam es zu einer Ausweitung des Engagements für diese Personengruppe (Huth 2019; Karakayali \& Kleist 2015).

- Verstärkte öffentliche Thematisierung von Umwelt- und Klimaschutz: In den letzten Jahren hat das Thema Umwelt- und Klimaschutz erhöhte mediale und politische Beachtung gefunden, nicht zuletzt angestoßen durch Umweltbewegungen wie Fridays for Future. Der Umwelt- und Klimaschutz ist eine der großen Herausforderungen für Politik und Gesellschaft und die Fragen zum Klimaschutz und zur Energiewende motivieren viele - vor allem junge Menschen - zum Engagement.

- Zunehmende Digitalisierung: Digitale Informations- und Kommunikationstechnologien haben in nahezu allen öffentlichen und privaten Lebensbereichen an Bedeutung gewonnen, auch im freiwilligen Engagement. Neue Engagementformen, die ausschließlich über das Internet stattfinden, sind entstanden, aber auch in bereits bestehenden Engagementstrukturen können Prozesse der Digitalisierung neue Zugangswege ermöglichen.

Festgestellt wird eine Pluralisierung und Individualisierung von Engagement (Mutz 2011; Schührer 2019) mit einer Zunahme von nicht vereinsförmig organisiertem und eher kurzfristig ausgeübtem Engagement, das eine hohe biografische Passung aufweisen muss. Zudem gibt es eine Abnahme der Kontinuität und eine Zunahme von episodenhaften freiwilligen Tätigkeiten in Form von kurzfristigen Projekten (Neufeind et al. 2015). Dies zeigt sich auch in den Daten der bisherigen Freiwilligensurvey-Erhebungen: Der Zeitumfang für die freiwillige Tätigkeit nimmt ab (Hameister et al 2017; siehe auch Kapitel 8); individuell organisiertes und informelles Engagement gewinnt gegenüber dem klassischen Engagement in Vereinen und Verbänden an Bedeutung (Simonson \& Vogel 2017; siehe auch Kapitel 9) und der Anteil der Engagierten, die im Rahmen ihrer freiwilligen Tätigkeit eine Leitungsfunktion übernehmen, sinkt (Hagen \& Simonson 2017; siehe auch Kapitel 9). Zugleich verändert die Digitalisierung 
das Engagement (Hinz et al. 2014). Für die Mehrheit der Engagierten stellt das Internet dabei bislang eine ergänzende Komponente zur Ausgestaltung der freiwilligen Tätigkeit dar: Nur ein geringer Anteil von Engagierten engagiert sich ausschließlich oder überwiegend im Internet (Hagen \& Simonson 2017; siehe auch Kapitel 11).

\subsection{Neue Themen im Freiwilligensurvey 2019}

Die Befragung zum freiwilligen Engagement in Deutschland bedarf einer gewissen Kontinuität, um Veränderungen in der Engagementlandschaft darlegen zu können. Diese Veränderungen erfordern jedoch auch, dass die Befragung des Freiwilligensurveys kontinuierlich angepasst wird, um neue Themen und auch neue Formen freiwilligen Engagements erfassen zu können. Im Zuge des gesellschaftlichen Wandels sind Veränderungen der öffentlichen Diskurse um das Engagement und des öffentlichen Interesses am Engagement festzustellen. Im Freiwilligensurvey wird dieses veränderte Interesse anhand folgender neu aufgenommener Themengebiete berücksichtigt:

- Einstellungen zur Demokratie, Vertrauen in staatliche Institutionen, politische Partizipation. Einige Studien stellen eine Vertrauenskrise hinsichtlich der Demokratie in Deutschland fest (zum Beispiel Decker et al. 2019). Auch vor dem Hintergrund einer verstärkten Mobilisierung politscher Bewegungen mit antidemokratischen Tendenzen wie bei der ,Identitären Bewegung' oder Pegida (Schumann 2018; Pfahl-Traughber 2019: 177 ff.) stellt sich verstärkt die Frage nach den demokratischen Einstellungen von Engagierten und ihrem Vertrauen in die Institutionen des demokratischen Systems. Neu aufgenommen in den Freiwilligensurvey 2019 wurden daher Fragen zu Demokratieeinstellungen und zum Vertrauen in staatliche Institutionen; die Abfrage zur politischen Partizipation wurde angepasst und erweitert, sodass die Verknüpfung von freiwilligem Engagement mit politischer Partizipation (siehe Kapitel 13) sowie von Engagement mit Demokratieeinstellungen und Institutionenvertrauen (siehe Kapitel 14) beleuchtet werden kann.

- Engagement für Geflüchtete und Engagement von Geflüchteten. Vor dem Hintergrund des deutlich gestiegenen Zuzugs geflüchteter Menschen nach Deutschland hat sich die Zahl der Studien zum Themengebiet Flucht und Engagement in den letzten Jahren deutlich erhöht. Allerdings steht hier meist das Engagement für Geflüchtete im Mittelpunkt (siehe zum Beispiel Karakayali \& Kleist 2015). Über das Engagement von Geflüchteten, das für diese eine Brückenfunktion in die Gesellschaft einnehmen kann (Speth 2018: 4), ist dagegen deutlich weniger bekannt. Im Freiwilligensurvey 2019 wurden neue Fragen für beide Perspektiven - Engagement für Geflüchtete und Engagement von Geflüchteten - aufgenommen. Bezüglich des Engagements für Geflüchtete gibt es zwei neue Fragen: Zum einen wird retrospektiv erfasst, ob sich Personen in den letzten fünf Jahren für Geflüchtete engagiert haben. Zum anderen wird erstmals erfasst, ob sich die aktuell ausgeübte 
freiwillige Tätigkeit von engagierten Personen an geflüchtete Menschen richtet (siehe Kapitel 10). Um das Engagement von Geflüchteten abzubilden, werden Personen, die nach Deutschland eingewandert sind, im Freiwilligensurvey 2019 erstmals gefragt, ob sie dies als Geflüchtete beziehungsweise Asylsuchende getan haben (siehe Kapitel 4).

- Formen der Internetnutzung. Die Digitalisierung der Gesellschaft eröffnet Potenziale für das Engagement und kann neue Engagementformen hervorbringen (Hinz et al. 2014). Um die Vielfalt der Internetnutzung im Engagement abzubilden, wird im Freiwilligensurvey 2019 die Internetnutzung im Rahmen der freiwilligen Tätigkeit erstmals differenziert anhand von fünf Formen erfragt: Beteiligung an sozialen Netzwerken oder Blogs, Website-Betreuung für die Organisation, Weitergabe von Informationen in Form von Newslettern oder Online-Berichten, Lehr- und Beratungstätigkeiten, die über das Internet angeboten werden, sowie Einwerbung von Spenden oder Engagierten über das Internet (siehe Kapitel 11).

- Zwecke von Geldspenden. Geldspenden sind ebenso wie das freiwillige Engagement eine wichtige Form zivilgesellschaftlichen Handelns (Hameister \& Vogel 2017; Priller \& Schupp 2011). Im Freiwilligensurvey wird 2019 erstmals ermittelt, welchen Zwecken die geleisteten Geldspenden zugutekommen. In Anlehnung an die Unterscheidung des Deutschen Spendenrates (Deutscher Spendenrat e. V. \& GFK 2019) werden soziale und humanitäre Hilfen (Spenden für den kirchlichen oder religiösen Bereich, für Kinder und Jugendliche, für die Not- und Katastrophenhilfe, für die Flüchtlingshilfe oder die Behinderten- oder Krankenhilfe), Spenden für die Entwicklungshilfe sowie Spenden für Sport und Bewegung, die Kultur- und Denkmalpflege sowie den Umwelt-, Natur- oder Tierschutz erfasst (siehe Kapitel 7).

\subsection{Vielfalt und Ungleichheit im Engagement}

Engagement ist nicht nur hinsichtlich der Inhalte und Formen der freiwilligen Tätigkeiten vielfältig, Vielfalt findet sich auch bei den freiwillig Engagierten selbst. Ganz unterschiedliche Menschen sind freiwillig engagiert: Menschen unterschiedlichen Geschlechts, Menschen unterschiedlichen Alters und mit unterschiedlicher Bildung, Menschen mit und ohne Migrationshintergrund. Diese Unterschiede können sich auch in Ungleichheiten im Zugang zum freiwilligen Engagement niederschlagen. Freiwilliges Engagement ist voraussetzungsvoll: Eine Person muss beispielsweise genügend freie Zeit zur Verfügung haben, sie muss die (zusätzlichen) Anforderungen und Belastungen, die ein Ehrenamt beziehungsweise eine freiwillige Tätigkeit unter Umständen mit sich bringt, tragen können, und sie muss - je nach Aufgabengebiet - Kenntnisse oder Fähigkeiten mitbringen. Diese Faktoren, die die Aufnahme und Ausübung eines freiwilligen Engagements bestimmen, können je nach Lebenssituation und nach Bevölkerungsgruppe deutlich variieren. 
- Geschlecht. Die Lebenssituation der Geschlechter unterscheidet sich in vielen Bereichen nach wie vor deutlich; zu denken ist beispielsweise an die weiterhin bestehende geschlechtsspezifische Arbeitsteilung. Diese unterschiedliche Arbeitsteilung kann sich auch auf die Beteiligung im freiwilligen Engagement auswirken. Die unterschiedliche zur Verfügung stehende Zeit kann nicht nur die Aufnahme eines freiwilligen Engagements beeinflussen, sondern auch, welche freiwilligen Tätigkeiten übernommen werden oder welcher zeitliche Aufwand für die freiwillige Tätigkeit eingesetzt werden kann.

- Altersgruppen. Unterschiedliche Altersgruppen spiegeln unterschiedliche Situationen im Lebensverlauf mit verschiedenen Anknüpfungspunkten und Möglichkeiten für Engagement wider. Es zeigen sich daher auch Unterschiede zwischen Altersgruppen im freiwilligen Engagement. Dies kann mit unterschiedlichen Zeitbudgets zusammenhängen, die für das Engagement zur Verfügung stehen, zum Beispiel aufgrund der jeweils unterschiedlich starken Einbindung in familiäre Aufgaben und in die Erwerbsarbeit, aber auch durch unterschiedliche Präferenzen hinsichtlich der Zeitgestaltung begründet sein. Menschen unterschiedlichen Alters bringen zudem auch die unterschiedlichsten Erfahrungswerte mit sich, die für das freiwillige Engagement günstig oder auch notwendig sein können.

- Bildungsgruppen. Bildung ist eine wichtige Ressource für freiwilliges Engagement; das spiegelt sich auch in der Beteiligung im freiwilligen Engagement von Menschen mit unterschiedlicher Bildung wider. Die Ursachen hierfür sind vielfältig: Die Möglichkeiten, sich über die Aufnahme und Ausübung eines freiwilligen Engagements $\mathrm{zu}$ informieren oder auch die eigenen Interessen $\mathrm{zu}$ vertreten, stehen in Zusammenhang mit der Bildung. Auch der Zusammenhang von Bildung, Einkommen und beruflicher Position wirkt sich auf die Beteiligung im freiwilligen Engagement aus, beispielsweise durch unterschiedliche Anknüpfungspunkte oder Zeitbudgets.

- Migrationshintergrund. Freiwilliges Engagement von Menschen mit Migrationshintergrund kann als Indikator für ihre gesellschaftlichen Teilhabechancen in der bundesdeutschen Gesellschaft verstanden werden. Da es sich bei der Gruppe der Menschen mit Migrationshintergrund um eine sehr heterogene Gruppe handelt, wird im Freiwilligensurvey nicht nur betrachtet, ob eine Person einen Migrationshintergrund hat oder nicht, sondern auch die eigene Zuwanderungserfahrung und die deutsche Staatsbürgerschaft werden für die Betrachtung freiwilligen Engagements von Menschen mit Migrationshintergrund herangezogen. ${ }^{3}$

3 Im vorliegenden Bericht verwenden wir, wie auch im letzten Bericht zum Freiwilligensurvey, das auf der Definition des Statistischen Bundesamts (Statistisches Bundesamt 2019) basierende Konzept des Migrationshintergrunds. Es handelt sich dabei um ein eingeführtes und weitverbreitetes Konzept, dass jedoch auch kritisch diskutiert wird. Die von der Bundesregierung eingesetzte Fachkommission zu den Rahmenbedingungen der Integrationsfähigkeit empfiehlt beispielsweise, auf den Begriff Migrationshintergrund zu verzichten und stattdessen von Eingewanderten und ihren (direkten) Nachkommen zu sprechen. Damit würde eine Fokussierung auf die erste und zweite Generation von 
So können die unterschiedlichen Erfahrungen, die Menschen mit eigener Zuwanderungserfahrung in der Schule, im Beruf oder im gesellschaftlichen Umfeld gesammelt haben, das Verständnis von Engagement prägen oder auch den Zugang dazu beeinflussen. Die Staatsangehörigkeit kann ebenfalls direkte Auswirkungen auf die Möglichkeiten der Engagementaufnahme haben, da beispielsweise ein Schöffenamt an die deutsche Staatsangehörigkeit geknüpft ist.

Im Bericht werden die für die Beteiligung und Ausgestaltung im freiwilligen Engagement zentralen Kriterien Geschlecht, Altersgruppen, Bildungsgruppen und Migrationshintergrund als Differenzierungsmerkmale betrachtet (für eine ausführliche Darstellung dieser Differenzierungsmerkmale siehe Kapitel 2). In Kapitel 4 werden darüber hinaus weitere (dort näher erläuterte) Differenzierungskriterien verwendet, um das Engagement unterschiedlicher Bevölkerungsgruppen darzustellen.

\subsection{Methodische Kontinuität und Weiterentwicklung des Freiwilligensurveys}

Für eine Sozialberichterstattung, die das Ziel hat, den sozialen Wandel abzubilden, ist es unabdingbar, dass die methodische Anlage von Studien über die Zeit hinweg konstant bleibt. Um etwa die Entwicklung von Engagementquoten im Zeitverlauf zu beobachten, ist es notwendig, dass die Fragen, mit denen freiwilliges Engagement erhoben wird, zu allen Befragungszeitpunkten vergleichbar sind. Nur dann kann zuverlässig Auskunft darüber gegeben werden, ob sich der Anteil der Bevölkerung verändert, der sich freiwillig engagiert. Ähnliches gilt für die Art der Gewinnung von Personen, die an der Befragung teilnehmen, sowie für statistische Verfahren, die dazu dienen, Aussagen über die Bevölkerung insgesamt zu treffen. Beide Verfahren sollten stabil gehalten werden, um Vergleiche über Zeitpunkte hinweg zu ermöglichen. Methodische Veränderungen sind aber dann notwendig, wenn sich zeigt, dass bisherige Verfahren der Verbesserung bedürfen, um den hohen methodischen Ansprüchen der Sozialberichterstattung zu genügen.

Der Freiwilligensurvey zielt darauf ab, die Situation des freiwilligen Engagements in Deutschland möglichst genau zu beschreiben. Voraussetzung dafür ist, dass die im Rahmen des Freiwilligensurveys befragten Personen die Bevölkerung in Deutschland gut abbilden - der Freiwilligensurvey soll repräsentativ für die Bevölkerung sein. Obwohl alle Stichproben des Freiwilligensurveys von 1999 bis 2019 sehr groß waren, bilden sie die Grundgesamtheit nicht vollständig ab, zum Beispiel weil nicht alle Bevölkerungsgruppen gleichermaßen gut erreichbar sind und bereit an Umfragen teil-

Eingewanderten erfolgen und die Aspekte Migration und Staatsangehörigkeit wären voneinander entkoppelt (Fachkommission der Bundesregierung zu den Rahmenbedingungen der Integrationsfähigkeit 2020). 
zunehmen. Daher werden die Daten gewichtet. Die Gewichtung zielt darauf ab, die Repräsentativität zu verbessern, um verallgemeinerbare Aussagen treffen zu können und die Grundgesamtheit möglichst gut abzubilden (Kiesl 2019). Verzerrende Faktoren der Stichprobenzusammensetzung (zum Beispiel eine disproportionale Ziehung nach Regionen) sowie ungleiche Teilnahmewahrscheinlichkeiten (zum Beispiel nach Alter) können durch Gewichtung ausgeglichen werden. Im Rahmen des Freiwilligensurveys wurden bisher die Gewichtungsmerkmale Bundesland, Gemeindegrößenklasse (BIK), Geschlecht und Altersgruppe herangezogen (Schiel et al. 2020). Diese Gewichtung wird nun um das Gewichtungsmerkmal der schulischen Bildung ergänzt. Personen aus unterschiedlichen Bildungsgruppen nehmen mit unterschiedlicher Wahrscheinlichkeit an Umfragen teil; daher sind verschiedene Bildungsgruppen in den Daten über- beziehungsweise unterrepräsentiert. Insbesondere Personen mit höherer Bildung sind eher bereit, an Befragungen teilzunehmen als Personen mit niedrigerer Bildung (Engel \& Schmidt 2019). Da Menschen mit höherer Bildung anteilig häufiger freiwillig engagiert sind als Menschen mit niedriger Bildung, wurden durch die Nicht-Berücksichtigung der Bildung in der Datengewichtung in den bisherigen Berichten zum Freiwilligensurvey die Engagementquoten zwischen 1999 und 2014 überschätzt.

Obwohl das Problem der bildungsbezogenen Stichprobenverzerrung seit der ersten Befragung des Freiwilligensurveys im Jahr 1999 besteht, wurde es zum ersten Mal im Zuge des Berichts zur Erhebung 2014 diskutiert. Dabei wurden die bildungsgewichteten Engagementquoten den nicht bildungsgewichteten Engagementquoten gegenübergestellt (Simonson et al. 2017: 76; siehe auch Kapitel 2). Im Jahr 2019 wird das Vorgehen bei der Gewichtung des Freiwilligensurveys angepasst: Es wird zum ersten Mal durchgängig auch nach Bildung gewichtet, also zusätzlich zu den bisherigen Gewichtungsmerkmalen auch die schulische Bildung als Gewichtungsfaktor einbezogen. Durch den Einbezug der schulischen Bildung in die Gewichtung wird die Grundgesamtheit der Wohnbevölkerung Deutschlands ab 14 Jahren nun besser abgebildet. Die Gewichtung inklusive schulischer Bildung wird in diesem Bericht auf die Daten aller fünf Erhebungswellen von 1999 bis 2019 angewendet. Die Engagementquoten fallen durch dieses Vorgehen für alle Erhebungswellen niedriger aus als die bisher ohne diese Gewichtung berechneten Quoten, und zwar um jeweils drei bis vier Prozentpunkte. Es ändert sich somit insgesamt das Niveau der Engagementquoten, der Trend bleibt jedoch im Wesentlichen bestehen. Die Gewichtung der Daten inklusive Bildung wirkt sich auch auf andere Ergebnisse aus.

Für eine bessere Abbildung der Bevölkerung in Deutschland werden weiterhin die methodischen Neuerungen beibehalten, die im Freiwilligensurvey 2014 getroffen wurden. So werden Interviews nicht nur über das Festnetz, sondern auch über Mobilfunknummern geführt. Das steigert die Wahrscheinlichkeit, Personen zu erreichen, die eine hohe Mobilität aufweisen und über das Festnetz schwer erreichbar, sind sowie von Personen, die über keinen Festnetzanschluss verfügen. Außerdem werden Interviews in deutscher Sprache, aber auch in fünf weiteren Sprachen geführt (Eng- 
lisch, Arabisch, Russisch, Türkisch und Polnisch). Dieses Vorgehen verbessert den Einbezug von Menschen mit Migrationshintergrund in den Freiwilligensurvey.

\subsection{Der Freiwilligensurvey 2019 und die Corona-Pandemie}

Nachdem sich im Januar 2020 erste Personen in Deutschland mit dem Coronavirus SARS-CoV-2 infiziert hatten und die Zahl der Erkrankten immer weiter anstieg, wurde die Pandemie Mitte März 2020 durch Regeln zu ihrer Eindämmung wie Lockdown und Kontaktbeschränkungen für die breite Bevölkerung spürbar. Mit dem zweiten Lockdown, der im Oktober 2020 beschlossen und ab Mitte Dezember 2020 in verschärfter Form bis ins Jahr 2021 hinein verlängert wurde, ist abzusehen, dass die Corona-Pandemie mindestens auch das Jahr 2021 noch weiterhin prägen wird. Die Pandemie hatte und hat somit tiefgreifende Auswirkungen auf die Gesellschaft insgesamt und damit auch auf die Zivilgesellschaft sowie das freiwillige Engagement.

Der Fünfte Deutsche Freiwilligensurvey wurde im Jahr 2019 erhoben. Die telefonische Befragung von mehr als 27.000 Menschen ab 14 Jahren in Deutschland fand im Zeitraum von März 2019 bis einschließlich November 2019 statt. Die Datenerhebung wurde also bereits deutlich vor dem Beginn der Corona-Pandemie abgeschlossen. Mit den Daten des Freiwilligensurveys 2019 lässt sich somit die Situation des freiwilligen Engagements in Deutschland vor der Corona-Pandemie darstellen. Allerdings ist es nicht möglich, mithilfe der Daten des Freiwilligensurveys 2019 Aussagen über die Auswirkungen der Corona-Pandemie auf das freiwillige Engagement zu treffen.

Eine zentrale Stärke des Freiwilligensurveys liegt in der langfristigen Berichterstattung und im Nachzeichnen größerer Entwicklungslinien im freiwilligen Engagement über einen Zeitraum von nunmehr zwei Jahrzehnten. Aufgrund der Fünfjahrestaktung des Surveys können unerwartet auftretende Ereignisse und damit einhergehende Veränderungen unter Umständen nicht oder nur mit Zeitverzug erhoben und berichtet werden.

Veränderungen im Engagement aufgrund der Corona-Pandemie lassen sich nur anhand weiterer Datenerhebungen nachzeichnen. Der Freiwilligensurvey 2019 kann hier als Vergleichsanker fungieren, um Aussagen über Veränderungen des freiwilligen Engagements zu ermöglichen und die Auswirkungen der Corona-Pandemie auf das freiwillige Engagement in Deutschland beurteilen zu können.

\subsection{Ausblick auf den Bericht}

Der Bericht zum Deutschen Freiwilligensurvey 2019 ist in vier Teile untergliedert: Teil I enthält neben diesem Einleitungskapitel eine Darstellung der Methodik des Freiwilligensurveys. In Teil II werden die Entwicklung des freiwilligen Engagements 
in Deutschland sowie Unterschiede und Ungleichheiten im Zugang zum freiwilligen Engagement von verschiedenen Bevölkerungsgruppen dargelegt. Teil III befasst sich mit der Ausgestaltung des freiwilligen Engagements. In Teil IV wird die Verknüpfung von politischer Partizipation, Einstellungen zur Demokratie und zu demokratischen Institutionen und Engagement beleuchtet. Der Bericht schließt mit einer zusammenfassenden Gesamtschau und gesellschaftspolitischen Einordnung der Ergebnisse.

\section{Literatur}

Alscher, M., Priller, E., \& Burkhardt, L. (2021). Zivilgesellschaftliches Engagement. In: Statistisches Bundesamt (Destatis), Wissenschaftszentrum Berlin für Sozialforschung (WZB) \& Bundesinstitut für Bevölkerungsforschung (BiB) (Hrsg.) Datenreport 2021. Ein Sozialbericht für die Bundesrepublik Deutschland (S. 399-407). Bonn: Bundeszentrale für politische Bildung.

Blaeschke, F., \& Freitag, H.-W. (2021). Bildungsbeteiligung, Bildungsniveau und Bildungsbudget. In: Statistisches Bundesamt (Destatis), Wissenschaftszentrum Berlin für Sozialforschung (WZB) \& Bundesinstitut für Bevölkerungsforschung (BiB) (Hrsg.) Datenreport 2021. Ein Sozialbericht für die Bundesrepublik Deutschland (S. 101-120). Bonn: Bundeszentrale für politische Bildung.

Decker, F., Best, V., Fischer, S., \& Küppers, A. (2019). Vertrauen in Demokratie. Wie zufrieden sind die Menschen in Deutschland mit Regierung, Staat und Politik? Berlin: Friedrich-EbertStiftung.

Deutscher Bundestag (2002). Bericht der Enquete-Kommission „Zukunft des Bürgerschaftlichen Engagements“. Bürgerschaftliches Engagement: auf dem Weg in eine zukunftsfähige Bürgergesellschaft (Drucksache 14/8900). Berlin: Deutscher Bundestag.

Deutscher Bundestag (2017). Zweiter Gleichstellungsbericht (Bundestagsdrucksache 18/12840). Berlin: Deutscher Bundestag.

Deutscher Spendenrat e. V. \& GFK (2019). Bilanz des Helfens 2018. Berliner Pressekonferenz, 28. Februar 2017. Online: www.spendenrat.de/wp-content/uploads/2019/o2/Bilanz_des_Helfens_2019.pdf (zuletzt abgerufen 12.11. 2019).

Ebert, O. (2018). Engagiert für Demokratie - Herausforderung und Perspektiven in Ostdeutschland. BBE-Newsletter Nr. 24. Online: https://www.b-b-e.de//fileadmin/Redaktion/o5_ Newsletter/o1_BBE_Newsletter/2018/newsletter-24-ebert.pdf (zuletzt abgerufen 10. 03. 2021).

Engel, U., \& Schmidt, B.-O. (2019). Unit- und Item-Nonresponse. In: N. Baur \& J. Blasius (Hrsg.) Handbuch Methoden der empirischen Sozialforschung (S. 385-404). Wiesbaden: Springer VS.

Fachkommission der Bundesregierung zu den Rahmenbedingungen der Integrationsfähigkeit (2020). Gemeinsam die Einwanderungsgesellschaft gestalten. Bericht der Fachkommission der Bundesregierung zu den Rahmenbedingungen der Integrationsfähigkeit. Online: https://www. fachkommission-integrationsfaehigkeit.de/resource/blob/1786706/1787474/5a5d62f9636b87f1 ofdoe271ba326471/bericht-de-data.pdf?download=1 (zuletzt abgerufen 03. 03. 2021).

Geißel, B. (2006). Kritische Bürgerinnen und Bürger - eine Gefahr für Demokratien? Aus Politik und Zeitgeschichte (APuZ), (12), 3-9. 
Hagen, C., \& Simonson, J. (2017). Inhaltliche Ausgestaltung und Leitungsfunktionen im freiwilligen Engagement. In: J. Simonson, C. Vogel \& C. Tesch-Römer (Hrsg.) Freiwilliges Engagement in Deutschland - Der Deutsche Freiwilligensurvey 2014 (S. 299-331). Wiesbaden: Springer VS.

Hameister, N., Müller, D., \& Ziegelmann, J.P. (2017). Zeitlicher Umfang, Häufigkeit und biografische Dauer des freiwilligen Engagements. In: J. Simonson, C. Vogel \& C. Tesch-Römer (Hrsg.) Freiwilliges Engagement in Deutschland - Der Deutsche Freiwilligensurvey 2014 (S. 333354). Wiesbaden: Springer VS.

Hameister, N., \& Vogel, C. (2017). Geldspenden, Spendenbeträge und freiwilliges Engagement. In: J. Simonson, C. Vogel \& C. Tesch-Römer (Hrsg.) Freiwilliges Engagement in Deutschland Der Deutsche Freiwilligensurvey 2014 (S. 217-234). Wiesbaden: Springer VS.

Hartnuß, B., Olk, T., \& Klein, A. (2011). Engagementpolitik. In: T. Olk \& B. Hartnuß (Hrsg.) Handbuch Bürgerschaftliches Engagement (S. 761-776). Weinheim: Beltz Juventa.

Hinz, U., Wegener, N., Weber, M., \& Fromm, J. (2014). Digitales Bürgerschaftliches Engagement. Berlin: FOKUS. Online: https://www.oeffentliche-it.de/documents/10181/14412/Digitales $+\mathrm{B} \% \mathrm{C}_{3} \% \mathrm{BCrgerschaftliches+Engagement} \mathrm{(zuletzt} \mathrm{abgerufen} \mathrm{11.} \mathrm{02.} \mathrm{2021).}$

Huth, S. (2019). Die Rolle von Migrantenorganisationen im Flüchtlingsbereich. Bestandsaufnahme und Handlungsempfehlungen. Frankfurt am Main: INBAS-Sozialforschung GmbH (INBAS).

Karakayali, S., \& Kleist, J. O. (2015). EFA-Studie: Strukturen und Motive der ehrenamtlichen Flüchtlingsarbeit (EFA) in Deutschland. 1. Forschungsbericht: Ergebnisse einer explorativen Umfrage vom November/Dezember 2014. Berlin: Berliner Institut für empirische Integrations- und Migrationsforschung (BIM), Humboldt-Universität zu Berlin.

Kausmann, C., Burkhardt, L., Rump, B., Kelle, N., Simonson, J., \& Tesch-Römer, C. (2019). Zivilgesellschaftliches Engagement. In: H. Krimmer (Hrsg.) Datenreport Zivilgesellschaft (S. 5591). Wiesbaden: Springer VS.

Kiesl, H. (2019). Gewichtung. In: N. Baur \& J. Blasius (Hrsg.) Handbuch Methoden der empirischen Sozialforschung (S. 405-412). Wiesbaden: Springer VS.

Klein, A., \& Müller B. (2016). Demokratische Mitte oder Bad Civil Society?. In: A. Zick, B. Küpper \& D. Krause (Hrsg.) Gespaltene Mitte - Feindselige Zustände (S. 185-201). Bonn: J.H. W. Dietz Nachf. GmbH.

Morrow-Howell, N., Hong, S.-I., \& Tang, F. (2009). Who Benefits From Volunteering? Variations in Perceived Benefits. The Gerontologist, 49(1), 91-102.

Mutz, G. (2011). Pluralisierung und Entgrenzung in der Erwerbsarbeit, im Bürgerengagement und in der Eigenarbeit. Arbeit, 14(11), 21-32.

Neufeind, M., Güntert, S.T., \& Wehner, T. (2015). Neue Formen der Freiwilligenarbeit. In: T. Wehner \& S. T. Güntert (Hrsg.) Psychologie der Freiwilligenarbeit. Motivation, Gestaltung und Organisation (S. 195-220). Berlin, Heidelberg: Springer.

Pfahl-Traughber, A. (2019). Rechtsextremismus in Deutschland: Eine kritische Bestandsaufnahme. Wiesbaden: Springer VS. 
Priemer, J., Bischoff, A., Hohendanner, C., Krebstakies, R., Rump, B., \& Schmitt, W. (2019). Organisierte Zivilgesellschaft. In: H. Krimmer (Hrsg.) Datenreport Zivilgesellschaft (S. 7-54). Wiesbaden: Springer VS.

Priller, E., \& Schupp, J. (2011). Soziale und ökonomische Merkmale von Geld- und Blutspendern in Deutschland. DIW Wochenbericht, 78(29), 3-10.

Putnam, R. D. (2000). Bowling alone. The collapse and revival of American community. New York: Simon \& Schuster.

Rauch, A., Tisch, A., \& Tophoven, S. (2017). Erwerbsbeteiligung Älterer. In: J. Möller \& U. Walwei (Hrsg.): Arbeitsmarkt kompakt. Analysen, Daten, Fakten (S. 30-31). Bielefeld: W. Bertelsmann Verlag.

Roth, R. (2010). Engagementförderung als Demokratiepolitik: Berücksichtigung einer Reformbaustelle. In: T. Olk, A. Klein, \& B. Hartnuß (Hrsg.) Engagementpolitik. Die Entwicklung der Zivilgesellschaft als politische Aufgabe (S. 611-636). Wiesbaden: VS Verlag für Sozialwissenschaften.

Schiel, S., Ruiz Marcos, J., \& Fahrenholz, M. (2020). Methodenbericht. Deutscher Freiwilligensurvey 2019. Bonn: infas Institut für angewandte Sozialwissenschaft.

Schührer, A. K. (2019). Migration und Engagement. Wiesbaden: Springer VS.

Schumann, F. (2018). Antidemokratische Demokratiekritik: Zum Demokratieverständnis politischer Empörungsbewegungen. Jena: Institut für Demokratie und Zivilgesellschaft. Online: https://www.idz-jena.de/wsddet/antidemokratische-demokratiekritik-zum-demokratieverstaendnis-politischer-empoerungsbewegungen/ (zuletzt abgerufen 29. 08. 2019).

Sellach, B., \& Libuda-Köster, A. (2017). Gleichstellungspolitik im Spiegel der Zeitverwendungserhebung. Ein Vergleich der Ergebnisse der Zeitverwendungserhebungen von 2001/2002 und 2012/2013. In: Statistisches Bundesamt (Hrsg.) Wie die Zeit vergeht - Analysen zur Zeitverwendung in Deutschland. Beiträge zur Ergebniskonferenz der Zeitverwendungserhebung 2012/13 am 5./6. Oktober 2016 in Wiesbaden (S. 25-44). Wiesbaden: Statistisches Bundesamt.

Simonson, J., Hameister, N., \& Vogel, C. (2017). Daten und Methoden des Deutschen Freiwilligensurveys. In: J. Simonson, C. Vogel \& C. Tesch-Römer (Hrsg.) Freiwilliges Engagement in Deutschland - Der Deutsche Freiwilligensurvey 2014 (S. 51-88). Wiesbaden: Springer VS.

Simonson, J., \& Vogel, C. (2017). Organisationale Struktur des freiwilligen Engagements und Verbesserungsmöglichkeiten der Rahmenbedingungen. In: J. Simonson, C. Vogel \& C. TeschRömer (Hrsg.) Freiwilliges Engagement in Deutschland - Der Deutsche Freiwilligensurvey 2014 (S. 523-548). Wiesbaden: Springer VS.

Simonson, J., \& Vogel, C. (2020). Freiwilliges Engagement im Alter. In: K. R. Schroeter, C. Vogel \& H. Künemund (Hrsg.) Handbuch Soziologie des Alter(n)s. Springer Reference Sozialwissenschaften. Wiesbaden: Springer VS.

Speth, R. (2018). Engagiert in neuer Umgebung: Empowerment von geflüchteten Menschen zum Engagement. Arbeitspapier (Opuscula Nr. 108). Berlin: Maecenata Institut für Philanthropie und Zivilgesellschaft.

Statistisches Bundesamt (2019). Bevölkerung mit Migrationshintergrund - Ergebnisse des Mikrozensus 2018. Wiesbaden: Statistisches Bundesamt. 
Verba, S., Schlozman, K. L., \& Brady, H.E. (1995). Voice and equality: Civic voluntarism in American politics. Cambridge, MA: Harvard University Press.

Vogel, C., Wettstein, M., \& Tesch-Römer, C. (Hrsg.) (2019). Frauen und Männer in der zweiten Lebenshälfte. Älterwerden im sozialen Wandel. Wiesbaden: Springer VS.

Wanger, S. (2017). Frauenerwerbstätigkeit. In: J. Möller \& U. Walwei (Hrsg.) Arbeitsmarkt kompakt. Analysen, Daten, Fakten (S. 27-29). Bielefeld: W. Bertelsmann Verlag.

Wilson, J. (2012). Volunteerism Research: A Review Essay. Nonprofit and Voluntary Sector Quarterly, 41(2), 176-211.

Open Access Dieses Kapitel wird unter der Creative Commons Namensnennung Nicht kommerziell 4.0 International Lizenz (http://creativecommons.org/licenses/bync/4.0/deed.de) veröffentlicht, welche die nicht-kommerzielle Nutzung, Vervielfältigung, Bearbeitung, Verbreitung und Wiedergabe in jeglichem Medium und Format erlaubt, sofern Sie den/die ursprünglichen Autor(en) und die Quelle ordnungsgemäß nennen, einen Link zur Creative Commons Lizenz beifügen und angeben, ob Änderungen vorgenommen wurden.

Die in diesem Kapitel enthaltenen Bilder und sonstiges Drittmaterial unterliegen ebenfalls der genannten Creative Commons Lizenz, sofern sich aus der Abbildungslegende nichts anderes ergibt. Sofern das betreffende Material nicht unter der genannten Creative Commons Lizenz steht und die betreffende Handlung nicht nach gesetzlichen Vorschriften erlaubt ist, ist auch für die oben aufgeführten nicht-kommerziellen Weiterverwendungen des Materials die Einwilligung des jeweiligen Rechteinhabers einzuholen. 